\title{
Vascularization of repaired limb bone defects using chitosan- $\beta$-tricalcium phosphate composite as a tissue engineering bone scaffold
}

\author{
LE YANG $^{1}$, QINGHUA WANG ${ }^{2}$, LIHUA PENG $^{3}$, HONG YUE $^{4}$ and ZHENDONG ZHANG $^{5}$
}

\author{
${ }^{1}$ Saint Petersburg State I.P. Pavlov Medical University, St. Petersburg 197101, Russia; ${ }^{2}$ Department of Orthopedics, \\ Hukou County Hospital of Traditional Chinese Medicine, Hukou, Jiangxi 332500; ${ }^{3}$ Department of Orthopedics, \\ The People's Hospital of Bishan County, Bishan, Chongqing 402760; ${ }^{4}$ Department of Orthopedics, \\ Zibo Zhangdian Hospital of Traditional Chinese Medicine, Zibo, Shandong 255035; \\ ${ }^{5}$ Department of Orthopedics, The 301 Military Hospital, \\ Beijing 100853, P.R. China
}

Received October 29, 2013; Accepted July 21, 2014

DOI: $10.3892 / \mathrm{mmr} .2015 .3653$

\begin{abstract}
Ensuring histocompatibility in the tissue engineering of bones is a complex issue. The aim of this study was to observe the feasibility of chitosan- $\beta$-tricalcium phosphate composite in repairing limb bone defects, and to evaluate the therapeutic effects on osteogenesis. Beagle mesenchymal stem cells (MSCs) were divided into an experimental group that was cultured with an injectable form of chitosan- $\beta$-tricalcium phosphate composite and a control group. The effect of the composite on bone tissue growth was evaluated by MTT assay. In addition, 12-month-old beagles were subjected to $15-\mathrm{mm}$ femur defects and subsequently implanted with scaffolds to observe the effects on osteogenesis and vascularization. The dogs were subdivided into two groups of five animals: Group A, which was implanted with scaffold-MSC compounds, and Group B, which was implanted with scaffolds alone. The dogs were observed on the 2 nd, 4 th, 8 th and 12 th weeks post-implantation. Scanning electron microscopy analysis revealed that the composite was compatible with MSCs, with similar outcomes in the control and experimental groups. MTT analysis additionally showed that the MSCs in the experimental group grew in a similar manner to those in the control group. The composite did not significantly affect the MSC growth or proliferation. In combination with MSCs, the scaffold materials were effective in the promotion of osteogenesis and vascularization. In conclusion, the chitosan- $\beta$-tricalcium phosphate
\end{abstract}

Correspondence to: Professor Qinghua Wang, Department of Orthopedics, Hukou County Hospital of Traditional Chinese Medicine, 39 Shizhongshan Road, Hukou, Jiangxi 332500, P.R. China E-mail: wangqinghuado@163.com

Key words: chitosan- $\beta$-tricalcium phosphate composite, injectable tissue engineering bone, scaffold composite was compatible with the MSCs and did not affect cellular growth or proliferation, therefore proving to be an effective injectable composite for tissue engineered bone. Simultaneous implantation of stem cells with a carrier composite proved to function effectively in the repair of bone defects.

\section{Introduction}

At present, tissue engineered bones can be prepared, according to the specific requirements of the host, through the implantation of tissues and materials with the desirable physiological functions required to fix and repair bone defects (1). This technique has attracted significant focus, as it is a minimally invasive, easily performed procedure that maintains the blood supply to the defective area. Currently, there are two main types of scaffolds, natural bio-derived materials (e.g. collagen, coral and bio-derived bones), and synthetic materials (e.g. bioceramics and polymer materials). As only one type of material is incapable of meeting the requirements of bone tissue repair, composite scaffold materials are preferred (2). However, histocompatibility remains problematic $(3,4)$. Therefore, a novel injectable chitosan- $\beta$-tricalcium phosphate composite was used in the present study to act as a carrier for mesenchymal stem cells (MSCs). The compatibility of the MSCs with the composite scaffold and the subsequent effects on cellular proliferation were analyzed. The results of this study may provide valuable evidence for the preparation of analogous injectable tissue engineered bones.

\section{Materials and methods}

General information. MSCs (China Infrastructure of Cell Line Resources, Beijing, China) were divided into two groups: An experimental study group that was cultured with injectable chitosan- $\beta$-tricalcium phosphate composite and a control group that was cultured with $\beta$-tricalcium phosphate composite 
only. The growth of the cells and tissues was verified by MTT assay to determine the effects of the composite. In addition, 10 12-month-old beagles were implanted with composite scaffolds and subsequently observed for the effects on osteogenesis and vascularization. The dogs were subsequently divided into Groups A and B ( $n=5 /$ group). Group A was subjected to $15-\mathrm{mm}$ defects on the femur and was then was implanted with scaffolds carrying MSCs. Group B was implanted with the composite scaffold materials alone, without MSCs. The two groups were observed on the 2nd, 4th, 8th and 12th weeks post-implantation (Fig. 1).

Sampling site and criteria. The experiments were performed in the Experimental Center of Hukou County Hospital of Traditional Chinese Medicine (Hukou, China). Healthy 12-month-old beagles weighing 8-10 kg were purchased from the Experimental Animal Center of Shandong University (Jinan, China). The dogs were free of infections or genetic diseases, and without history of limb bone fracture. The present study was approved by the Ethics Committee of The Saint-Petersburg State I.P. Pavlov Medical University (St. Petersburg, Russia).

Cell culture. MSCs were cultured under sterile conditions, in Dulbecco's modified Eagle's medium containing high-glucose levels as a carbon source and serum in 10-20\% $\mathrm{CO}_{2}$. MSCs were extracted from the animals by puncture under anesthesia. Bone marrow $(10 \mathrm{ml})$ was collected using a 16-gauge bone needle, and then filtered and cultured at $37^{\circ} \mathrm{C}$ in $10-20 \% \mathrm{CO}_{2}$. The culture medium was refreshed every three days until the end of the primary culture. Pancreatin enzyme was subsequently added to the culture medium in order to separate adhesive cells that were selectively cultured in $\alpha$-minimal essential medium supplemented with $10 \%$ fetal bovine serum, with alendronate sodium used as an inhibitor (Gibco-BRL, Gaithersburg, MD, USA). This new culture medium was also changed every three days (5-7). After three weeks of culture, the osteogenic differentiation of MSCs (third generation) was determined by positive alkaline phosphatase staining (Fig. 2)

Material preparation. Scaffold materials were prepared under sterile conditions at $37^{\circ} \mathrm{C}$ in an atmosphere containing $5 \%$ $\mathrm{CO}_{2}$, using chitosan and $\beta$-tricalcium phosphate as the liquid and solid material components, respectively (8). The mixture transformed from a liquid to solid state, and was then cut into $48-\mathrm{mm}^{3}$ sections using a sterile knife. The third-generation MSCs, culture medium and incised scaffold materials were transferred to 24-well plates (Fig. 3).

Construction of scaffolds. The composite material was cut into $12 \times 15 \mathrm{~mm}$ pieces, into which were injected third-generation MSCs. The compounds were then cultured under sterile conditions in $\mathrm{CO}_{2}$ at $37^{\circ} \mathrm{C}$ for $4-6 \mathrm{~h}$, and were subsequently covered with culture medium.

MTT assay. Culture medium was discarded from the culture plates, which were then washed with phosphate-buffered saline. The cultures were subsequently stained with MTT and universal buffer, and cultured in $5 \% \mathrm{CO}_{2}$ at $37^{\circ} \mathrm{C}$ for $5 \mathrm{~h}$.

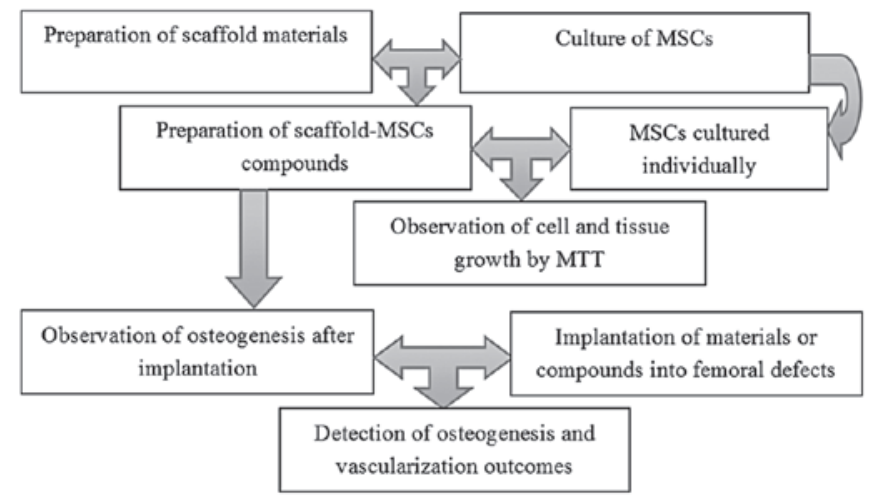

Figure 1. Experimental procedure. MSCs, mesenchymal stem cells.

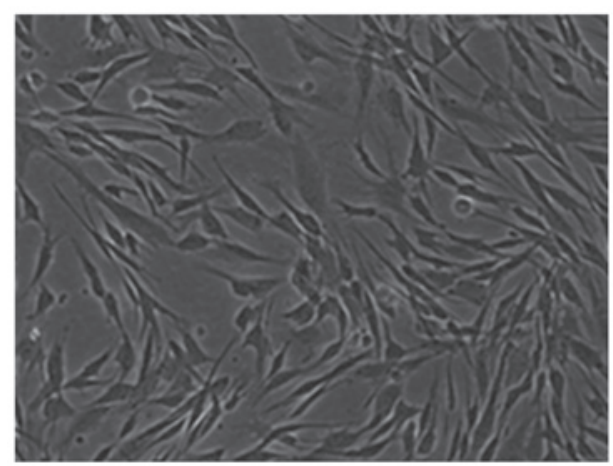

Figure 2. Mesenchymal stem cells stained by alkaline phosphatase (magnification, $\mathrm{x} 100)$

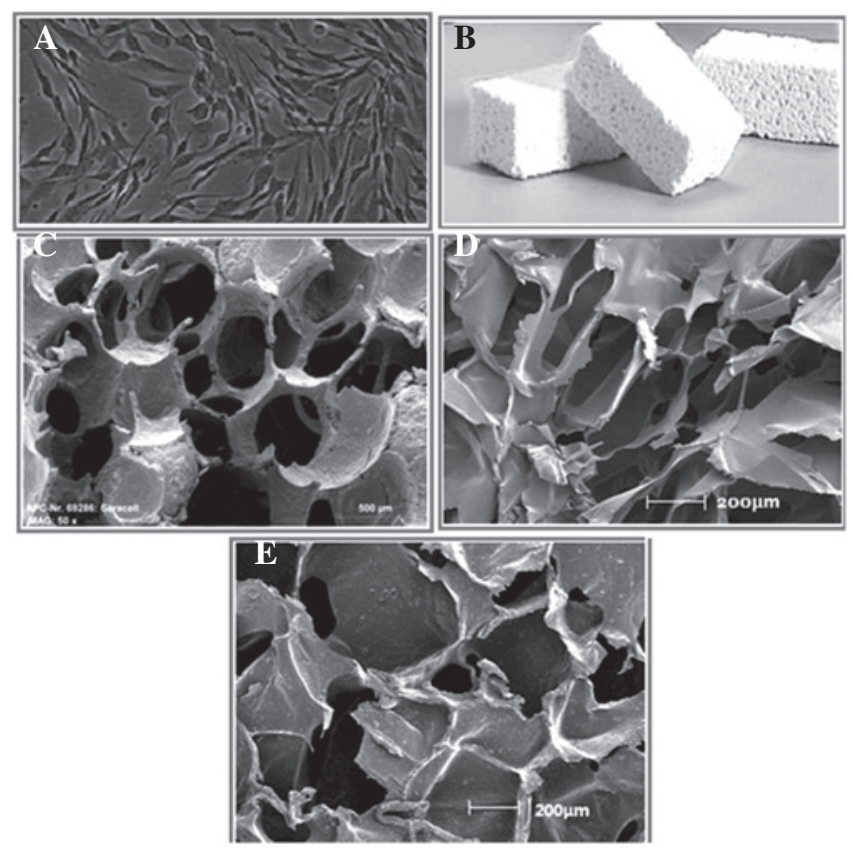

Figure 3. (A) Mesenchymal stem cells stained by alkaline phosphatase. (B) Chitosan- $\beta$-tricalcium phosphate composite. (C-E) Scanning electron microscopy images of: (C) Tricalcium phosphate; (D) chitosan; (E) chitosan-tricalcium phosphate composite (magnification, $\mathrm{x} 200$ ).

The MTT and buffer were discarded and $0.1 \mathrm{ml}$ acidified isopropanol was added to the plates and mixed by agitation for 
$15 \mathrm{~min}$. Finally, the absorbance of the products was measured at $570 \mathrm{~nm}$.

Scaffold implantation. The beagles were anesthetized by intravenous administration of $0.03 \mathrm{ml} / \mathrm{kg} 1 \%$ sodium pentobarbital, and the mucoperiosteum was separated following a skin incision $15 \mathrm{~mm}$ from the posterior limb. Femoral defects, $\sim 15 \mathrm{~mm}$ in length on one side of the femur, were prepared by an ultrasonic bone knife, followed by hemostasis. The tissue engineered bone scaffold materials were implanted, followed by suturing of the soft tissues, embedding and hemostasis. The wounds were rinsed with normal saline, sutured by layered closure and bandaged. Following the surgical procedure, the dogs were intramuscularly injected with 400,000 units penicillin for three consecutive days (qd). Under normal conditions, osteogenesis and vascularization would occur within 12 weeks.

Cluster of differentiation 31 (CD31) immunohistochemical staining. Histological analysis was performed using Masson's trichrome staining (Sigma-Aldrich, St. Louis, MO, USA). Sections were fixed at $60^{\circ} \mathrm{C}$ heat for $10 \mathrm{~min}$ and deparaffinized through ethanol hydration. The sections were subsequently sterilized with peroxidase and rinsed in buffer, prior to undergoing separation using trypsin and blocking with bovine serum albumin. The sections were then exposed to diluted anti-dog CD31 primary (1:100) and anti-goat secondary antibodies (Santa Cruz Biotechnology, Inc., Dallas, TX, USA), in order to perform double-antibody sandwich enzyme-linked immunosorbent assay. Upon clear color development, the reaction was terminated using distilled water. The sections were then double-stained with hematoxylin, prior to being differentiated, recovered, dehydrated, resealed and visualized under an inverted microscope. Areas of vascularization were stained.

Vascular casting method. Dogs were anesthetized by intravenous administration of $0.03 \mathrm{ml} / \mathrm{kg} 1 \%$ sodium pentobarbital. Skin incisions were then made through the subcutaneous tissues to expose the femoral arteries and veins for centrifugal intubation and fixation. Benzoyl peroxide phthalocyanine $(5 \mathrm{~g})$, dimethylaniline $(3 \mathrm{ml})$, dibutyl phthalate $(35 \mathrm{ml})$ and red oil paint (all Life Technologies, Carlsbad, CA, USA) were then added to $300 \mathrm{ml}$ pre-polymerized methyl methacrylate. Following the initiation of polymerization, blood vessels were infused until casting agents outflowed from the veins, which were then ligated. The infusion was continued until the peripheral skin was completely stained red $(250 \mathrm{ml}$ casting agent was used on each side); at this point, the infusion was terminated and the arteries were ligated. The infused methyl methacrylate monomers underwent complete polymerization following cryogenic storage of the blood vessels for 24-48 h while maintaining intravascular pressure, and the samples were preserved and fixed in $10 \%$ formaldehyde solution for 2-3 weeks.

Statistical analysis. All data were analyzed by SPSS 10.0 (SPSS Inc., Chicago, IL, USA). Groups were compared by a two-sample t-test to detect significant differences between the means of two samples and their totals. $\mathrm{P}<0.05$ was used to
Table I. Comparison between the MTT assay results.

\begin{tabular}{|c|c|c|c|c|}
\hline \multirow[b]{2}{*}{ Day } & \multicolumn{2}{|c|}{ Absorbance $^{a}$} & \multirow[b]{2}{*}{ t-test } & \multirow[b]{2}{*}{ P-value } \\
\hline & Group A & Group B & & \\
\hline 2nd & $0.118 \pm 0.032$ & $0.123 \pm 0.031$ & 0.550 & 0.384 \\
\hline 4 th & $0.367 \pm 0.064$ & $0.412 \pm 0.039$ & 2.941 & 0.247 \\
\hline 6th & $0.847 \pm 0.062$ & $0.911 \pm 0.089$ & 2.890 & 0.239 \\
\hline 8th & $1.128 \pm 0.084$ & $1.203 \pm 0.069$ & 3.380 & 0.084 \\
\hline
\end{tabular}

${ }^{a}$ Absorbance measured at $570 \mathrm{~nm}$. Data are presented as the mean \pm standard deviation.

Table II. Comparison between bone areas.

\begin{tabular}{lrrrr}
\hline & \multicolumn{2}{c}{ Bone area $\left(\mathrm{mm}^{2}\right)$} & & \\
\cline { 2 - 3 } Week & Group A & Group B & t-test & P-value \\
\hline 2nd & $3.5 \pm 0.8$ & $3.2 \pm 0.4$ & 1.64 & 0.213 \\
4th & $17.6 \pm 1.6$ & $6.8 \pm 1.2$ & 26.45 & 0.004 \\
8th & $50.4 \pm 6.3$ & $18.4 \pm 4.1$ & 20.85 & 0.007 \\
12th & $80.7 \pm 8.2$ & $51.2 \pm 6.1$ & 14.14 & 0.009 \\
\hline
\end{tabular}

Data are presented as the mean \pm standard deviation.

indicate a statistically significant difference. An independent $\mathrm{t}$-test was conducted using the following formula:

$$
\mathrm{t}=\frac{\overline{X 1}-\overline{X 2}}{\sqrt{\frac{(n 1-1) S 1^{2}+(n 2-1) S 2^{2}}{n 1+n 2-2}\left(\frac{1}{n 1}+\frac{1}{n 2}\right)}}
$$

Where $\mathrm{S} 1$ and $\mathrm{S} 2$ are two sample variances, and $\mathrm{n} 1$ and $\mathrm{n} 2$ are two sample sizes.

\section{Results}

MTT assay. The absorbances at $570 \mathrm{~nm}$ were measured on the $2 \mathrm{nd}, 4$ th, 6 th and 8 th days. The absorbances were lower in the experimental group than those in the control group (Table I). These results suggest that the experimental group had a higher proliferative capacity than the control group.

Osteogenesis outcomes following implantation. The scaffold-implanted areas were sampled 2, 4, 8 and 12 weeks after surgical intervention and the sections were subjected to Masson's trichrome staining. In the first two weeks, the degree of osteogenesis between the two groups was equivalent. After four weeks, Group A had significantly larger areas of newborn tissues as compared with Group B (Table II).

Vascularization outcomes following implantation. Sections were sampled at the sites of implantation 2, 4, 8 and 12 weeks 
Table III. Comparison between the number of blood vessels in the cross-sections.

\begin{tabular}{lcccc}
\hline & \multicolumn{2}{c}{ Blood Vessels (n) } & & \\
\cline { 2 - 3 } Week & Group A & Group B & t-test & P-value \\
\hline 2nd & $83.2 \pm 6.4$ & $26.4 \pm 4.6$ & 35.31 & 0.004 \\
4th & $254.8 \pm 8.4$ & $84.1 \pm 6.5$ & 78.73 & 0.002 \\
8th & $342.3 \pm 24.7$ & $126.1 \pm 16.4$ & 35.72 & 0.004 \\
12th & $512.4 \pm 36.6$ & $156.4 \pm 24.6$ & 39.55 & 0.004 \\
\hline
\end{tabular}

Data are presented as the mean \pm standard deviation.

after surgical intervention, and were subjected to CD31 staining to observe the number of new blood vessels. As shown in Figs. 4 and 5, Group A had a significantly higher number of new blood vessels than Group B.

The sections showed clear microvessels and blood vessels, which were reticulated along Haversian and Volkmann's canals. Nutrient vessels had extended into the endostea and periostea. In addition, Haversian canals were observed in the middle and internal and external lamellae, with blood vessels reticulating along Haversian and Volkmann's canals (Table III). Blood vessels were observed to have extended and grown well, and the differences between Groups A and $\mathrm{B}$ became more apparent with increased time.

\section{Discussion}

The injection of tissue engineered bones is a highly efficient intervention with minimal associated trauma. In the procedure, seed cells are carried in the liquid state, which then solidifies at the sites of bone defect following injection $(9,10)$.

Previous studies have endeavored to improve the histocompatibility of tissue engineering bones. Currently, only one type of material fails to satisfy the requirements of bone tissue repair, therefore composite scaffold materials are required $(11,12)$. Compared with previous studies, the present study presents the vascularization of repaired limb bone defects more clearly and directly. The findings suggest that chitosan- $\beta$-tricalcium phosphate composite is a suitable injectable tissue engineering bone material when stem cells are implanted simultaneously.

The currently available biomaterials are gels. These produce satisfactory osteogenesis but are low in stability and highly brittle, and thus perform poorly in repairing weight-bearing bones, such as femurs. At present, eligible tissue engineered bones are generally made from both organic or inorganic compounds, using citric acid as the crosslinking agent, thus fusing tissue cells well due to high strength and low toxicity (13-15).

To maintain the survival of tissue engineered bones in vivo, the vascularization and nerve generating capacities of the seeded cells should also be enhanced to accelerate bone recovery (16). The growth of seeded cells following implantation is associated with blood microcirculation. In cases of severe bone defect, the deep tissues may succumb

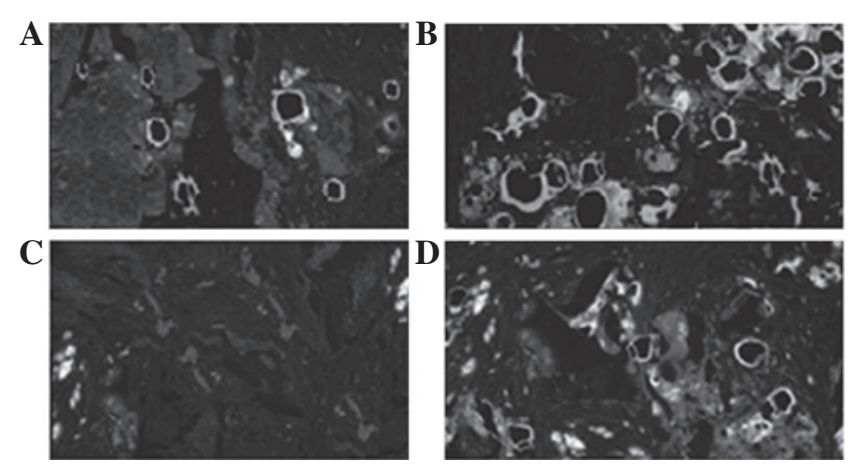

Figure 4. Cluster of differentiaion 31 immunohistochemical staining results 8 and 12 weeks after surgery. (A and B) Group A at (A) 8 and (B) 12 weeks. (C and D) Group B at (C) 8 and (D) 12 weeks.
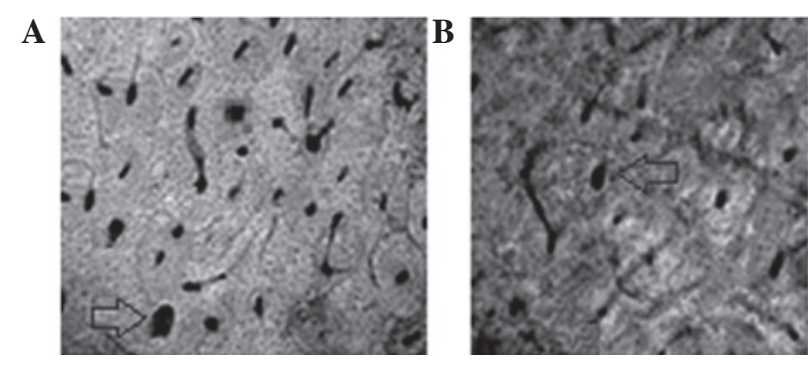

Figure 5. Vascular casting results: (A) Group A; (B) Group B. The arrow in each $100 \times 100-\mu \mathrm{m}$ cross-section of demineralized bone represents a vessel section filled with casting agents.

to ischemic necrosis. It is therefore essential to reconstruct the microcirculation to elevate the survival rate of seed cells. Periosteal tissue is abundant in nerves, of which a number enters the bone marrow together with nourishing blood vessels (17-20). The femur is supplied by the direct branches of blood vessels surrounding bone tissues as well as the periosteal vasoganglion. The vasoganglion forms with both skeletal muscles with arteries adhering to the bones and the periosteal branches adjoining to the arteries, and nutrients further penetrate into the tissues through pores in the bone.

Nervous tissue, which is widely distributed throughout bone tissue, controls the proliferation of osteoblasts and facilitates healing at the sites of defect upon bone fracture. Under normal conditions, nervous tissues regulate the metabolism of bone cells and therefore the nerves are considered to dominate the process of bone repair. Neurotransmitters secreted by nerve cells can upregulate or downregulate microcirculation and stimulate cell secretion. Peripheral nerves and the central nervous system cooperate in regulating the repair of defects $(21,22)$.

To date, liquid compounds have only been applicable to filling, rather than repairing, defects. Despite normal growth in liquid gels, it remains to be determined whether MSCs can grow in solid compounds (23). Reconstructing blood vessels and nervous tissues in defects not only recovers the blood supply and self-regulation of bones, but is also required for postoperative treatment and effective recovery. This study has therefore initiated a novel approach for the surgical repair of bone defects. 


\section{References}

1. Dimitriou R, Jones E, McGonagle D and Giannoudis PV: Bone regeneration: current concepts and future directions. BMC Med 9: 66, 2011.

2. Bose S, Roy M and Bandyopadhyay A. Recent advances in bone tissue engineering scaffolds. Trends Biotechnol 30: 546-554, 2012.

3. Muschler GF, Nakamoto C and Griffith LG: Engineering principles of clinical cell-based tissue engineering. J Bone Joint Surg Am 86-A: 1541-1558, 2004.

4. Muschler GF, Raut VP, Patterson TE, Wenke JC and Hollinger JO: The design and use of animal models for translational research in bone tissue engineering and regenerative medicine. Tissue Eng Part B Rev 16: 123-145, 2010.

5. Tasso R, Fais F, Reverberi D, Tortelli F and Cancedda R: The recruitment of two consecutive and different waves of host stem/progenitor cells during the development of tissue-engineered bone in a murine model. Biomaterials 31: 2121-2129, 2010

6. Yeatts $\mathrm{AB}$ and Fisher JP: Bone tissue engineering bioreactors: dynamic culture and the influence of shear stress. Bone 48: 171-181, 2011.

7. Yilgor P, Tuzlakoglu K, Reis RL, Hasirci N and Hasirci V: Incorporation of a sequential BMP-2/BMP-7 delivery system into chitosan-based scaffolds for bone tissue engineering. Biomaterials 30: 3551-3559, 2009.

8. Mouriño V and Boccaccini AR: Bone tissue engineering therapeutics: controlled drug delivery in three-dimensional scaffolds. J R Soc Interface 7: 209-227, 2010.

9. Holzwarth JM and Ma PX: Biomimetic nanofibrous scaffolds for bone tissue engineering. Biomaterials 32: 9622-9629, 2011.

10. Yamada Y, Nakamura S, Ito K, et al: Injectable bone tissue engineering using expanded mesenchymal stem cells. Stem Cells 31 : 572-580, 2013

11. Bi ZG, Han XG, Fu CJ, Cao Y and Yang CL. Reconstruction of large limb bone defects with a double-barrel free vascularized fibular graft. Chin Med J (Engl) 121: 2424-2428, 2008.

12. Noaman HH. Management of upper limb bone defects using free vascularized osteoseptocutaneous fibular bone graft. Ann Plast Surg 71: 503-509, 2013.
13. Jiang Q, Reddy N and Yang Y: Cytocompatible cross-linking of electrospun zein fibers for the development of water-stable tissue engineering scaffolds. Acta Biomater 6: 4042-4051, 2010.

14. Gyawali D, Nair P, Zhang Y, et al: Citric acid-derived in situ crosslinkable biodegradable polymers for cell delivery. Biomaterials 31: 9092-9105, 2010.

15. Kuo YC and Yeh CF: Effect of surface-modified collagen on the adhesion, biocompatibility and differentiation of bone marrow stromal cells in poly(lactide-co-glycolide)/chitosan scaffolds. Colloids Surf B Biointerfaces 82: 624-631, 2011.

16. Amini AR, Laurencin CT and Nukavarapu SP: Differential analysis of peripheral blood- and bone marrow-derived endothelial progenitor cells for enhanced vascularization in bone tissue engineering. J Orthop Res 30: 1507-1515, 2012.

17. Sun X, Su J, Bao J, et al: Cytokine combination therapy prediction for bone remodeling in tissue engineering based on the intracellular signaling pathway. Biomaterials 33: 8265-8276, 2012.

18. Zhao J, Luo C, Han W, Tu M, Zeng R, Cha Z and Zhou C: Fabrication and properties of poly(L-lactide)/hydroxyapatite/chitosan fiber ternary composite scaffolds for bone tissue engineering. J Polymer Eng 32: 283-289, 2012.

19. Pan L, Pei X, He R, Wan Q and Wang J: Multiwall carbon nanotubes/polycaprolactone composites for bone tissue engineering application. Colloids Surf B Biointerfaces 93: 226-234, 2012.

20. Higuita-Castro N, Gallego-Perez D, Pelaez-Vargas A, et al: Reinforced Portland cement porous scaffolds for load-bearing bone tissue engineering applications. J Biomed Mater Res B Appl Biomater 100B: 501-507, 2012.

21. Ji Y, Xu GP, Zhang ZP, Xia JJ, Yan JL and Pan SH: BMP-2/PLGA delayed-release microspheres composite graft, selection of bone participate diameters, and prevention of aseptic inflammation for bone tissue engineering. Ann Biomed Eng 38: 632-639, 2010

22. Clarke SA, Walsh P, Maggs CA and Buchanan F: Designs from the deep: marine organisms for bone tissue engineering. Biotechnol Adv 29: 610-617, 2011.

23. Alge DL, Bennett J, Treasure T, Voytik-Harbin S, Goebel WS and Chu TM: Poly(propylene fumarate) reinforced dicalcium phosphate dihydrate cement composites for bone tissue engineering. J Biomed Mater Res A 100: 1792-1802, 2012. 